\title{
Adolescents' neural response to social reward and real-world emotional closeness and positive affect
}

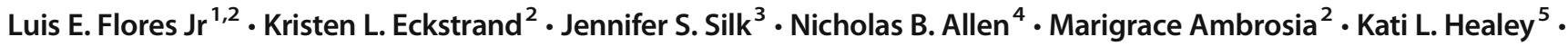 \\ Erika E. Forbes ${ }^{2}$
}

Published online: 25 June 2018

(C) This is a U.S. government work and its text is not subject to copyright protection in the United States; however, its text may be subject to foreign copyright protection 2018

\begin{abstract}
Feeling emotionally close to others during social interactions is a ubiquitous and meaningful experience that can elicit positive affect. The present study integrates functional magnetic resonance imaging (fMRI) and ecological momentary assessment (EMA) to investigate whether neural response to social reward (1) is related to the experience of emotional closeness and (2) moderates the association between emotional closeness and positive affect during and following social interactions. In this study, 34 typically developing adolescents (ages 14-18 years) completed a social-reward fMRI task, a monetary-reward fMRI task, and a 2-week EMA protocol regarding their social and affective experiences. Adolescents with greater right posterior superior temporal sulcus/ temporoparietal junction (pSTS/TPJ) response to social reward reported greater mean momentary emotional closeness. Neural response to social reward in the right pSTS/TPJ moderated how strongly momentary emotional closeness was associated with both concurrent positive affect and future peak happiness, but in different ways. Although emotional closeness had a significant positive association with concurrent positive affect among adolescents at both high and low right pSTS/TPJ response based on a follow-up simple slopes test, this association was stronger for adolescents with low right pSTS/TPJ response. In contrast, emotional closeness had a significant positive association with future peak happiness among adolescents with high right pSTS/TPJ response, but not among those with low right pSTS/TPJ response. These findings demonstrate the importance of neural response to social reward in key social processing regions for everyday experiences of emotional closeness and positive affect in the context of social interactions.
\end{abstract}

Keywords Social reward $\cdot$ Positive affect $\cdot$ Social interaction $\cdot$ Emotional closeness $\cdot$ Temporoparietal junction $\cdot$ Posterior superior temporal sulcus

Positive affect promotes well-being and protects against maladaptive outcomes, such as depression (Lyubomirsky, King, $\&$ Diener, 2005). Social interactions are meaningful and ubiquitous experiences that can elicit positive affect and engage reward circuitry (Kahneman, Krueger, Schkade, Schwarz, \& Stone, 2004); however, people differ in how they perceive,

Luis E. Flores, Jr

floresle@pitt.edu

1 VA Pittsburgh Healthcare System, MIRECC-Research Office Building (151R-U), University Drive C, Pittsburgh, PA 15240, USA

2 University of Pittsburgh School of Medicine, Pittsburgh, USA

3 University of Pittsburgh, Pittsburgh, USA

4 University of Oregon, Eugene, OR, USA

5 University of North Carolina, Chapel Hill, NC, USA respond to, and benefit from rewarding social interactions (Healey, Morgan, Musselman, Olino, \& Forbes, 2014). For instance, some may respond to pleasant interactions (e.g., receiving social approval) with heightened cognitive engagement and greater neural response in associated social circuitry, which may contribute to a greater positive affective response. Others might find the same experience much less pleasant or even aversive, which may not elicit significant positive affect. Thus, examining individual differences in neural response to social rewards may help elucidate how people differ in their experience of positive affect during everyday pleasant interactions.

\section{Social reward in adolescence}

Adolescence is a developmental period during which reward systems integrating behavior, brain function, and experience 
undergo dramatic changes in the service of developmental tasks such as exploration, learning about rewards, and individuation (Crone \& Dahl, 2012). For example, reward pursuit (Somerville, Jones, \& Casey, 2010) and responsivity (Ernst et al., 2005; Steinberg, 2008) peak in adolescence. Adolescents' activation in neural reward circuitry (e.g., increased ventral striatum, decreased medial prefrontal cortex $[\mathrm{mPFC}]$ ) differs from that in children and adults (e.g., Bjork et al., 2004; Ernst et al., 2005; Forbes et al., 2010). Alterations in neural reward circuitry in adolescence are related to potentially maladaptive outcomes, such as the development of substance use (Plichta \& Scheres, 2014), other impulsive behaviors (Hyman, Malenka, \& Nestler, 2006), and depression (Forbes \& Dahl, 2012). Differential neural and behavioral response to reward in adolescence also has a positive side, however, and is not necessarily maladaptive. Depending on the nature of the reward and the social context, such as maternal presence, greater ventral striatum reactivity can be related to prosocial and adaptive outcomes (e.g., family orientedness, less risk taking; Telzer, 2016). For instance, heightened ventral striatum reactivity to donating money to family is related to decreases in depression, whereas heightened ventral striatum reactivity to risky rewards is related to increases in depression (Telzer, Fuligni, Lieberman, \& Galván, 2014).

Most research on reward in adolescence has focused on nonsocial rewards (e.g., monetary reward). However, social contexts have important and potentially specific influences on brain and behavior. For instance, adolescents - compared with children and adults - are more likely to engage in risky behaviors (Steinberg, 2008) and exhibit greater ventral striatum activation in response to reward (Smith, Steinberg, Strang, \& Chein, 2015; Somerville et al., 2010) when in the presence of peers than when alone. In addition, adolescents have greater sensitivity and altered neural response to acceptance and rejection by peers compared with children and adults (Pfeifer \& Blakemore, 2012). Thus, social reward is an essential component of reward processing in adolescence. In fact, social rewards are arguably the most important class of reward during adolescence given that adolescence is characterized by a preoccupation with attaining social status, seeking friendships, and fostering intimacy with peers (Davey, Yücel, \& Allen, 2008). Alterations in response to social reward are associated with maladaptive outcomes, such as depression vulnerability (Monk et al., 2008; Olino, Silk, Osterritter, \& Forbes, 2015), social anhedonia (Healey et al., 2014), and social anxiety disorder (Richey et al., 2014). Social reward has been investigated by examining neural response to various stimuli, including standardized positive peer feedback (Davey, Allen, Harrison, Dwyer, \& Yücel, 2010; Guyer, Choate, Pine, \& Nelson, 2012; Silk et al., 2012), presentation of happy and/ or familiar faces (Lin, Adolphs, \& Rangel, 2012), provision of praise (Izuma, Saito, \& Sadato, 2008), and simulated increases in social status (Zink et al., 2008).

\section{Social brain network in adolescence}

Given the importance of social rewards in adolescence, the social brain network - which is a collection of brain regions that are involved in understanding others and social interactions (Blakemore, 2008; Burnett, Sebastian, Kadosh, \& Blakemore, 2011) - may play an important role in adolescents' positive affect and reward processing. Processes associated with the social brain network include mentalizing, which refers to understanding the mental states of others and is often used synonymously with theory of mind and perspective taking, and emotion regulation during social interactions. Key brain regions of the social brain network that are associated with mentalizing include the temporoparietal junction (TPJ), posterior superior temporal sulcus (pSTS), temporal poles, and medial prefrontal cortex (mPFC); those associated with emotion regulation in social interactions include the mPFC and ventrolateral prefrontal cortex (VLPFC; Burnett et al., 2011; Frith \& Frith, 2003). Changes in neural activation to mentalizing (Gunther Moor et al., 2012) and peer acceptance and rejection (Pfeifer \& Blakemore, 2012) occur over the course of adolescence. For instance, in the transition from adolescence to adulthood, there tends to be a linear decrease in $\mathrm{mPFC}$ activity and increase in pSTS/TPJ activity during mentalizing and social decision-making tasks, as well as a linear increase in VLPFC activity in response to social rejection (Burnett et al., 2011; Crone \& Dahl, 2012). These changes may reflect an increase in the use and ability to consider the thoughts of others in social situations and an increase in regulating negative emotion in response to social rejection as adolescents get older and transition into adulthood. Importantly, effective mentalizing and emotion regulation during social interactions improve social likeability (Gross \& John, 2003; Lopes, Salovey, Côté, Beers, \& Petty, 2005), which is a highly salient social reward in adolescence (Davey et al., 2008).

\section{Emotional closeness as a naturalistic social-affective experience}

Emotional closeness refers to feeling close and connected to others (i.e., the perception of emotional closeness). There have also been conceptualizations of emotional closeness that include objective components, such as engagement in emotionally intimate behaviors (e.g., disclosure of intimate thoughts, receipt of verbal and physical affection, the provision/receipt of emotional support; Flores \& Berenbaum, 2014). Importantly, emotional closeness is a key component of intimate relationships that helps fulfill the basic human need of belongingness (Baumeister \& Leary, 1995). During social interactions, feelings of closeness are associated with having heightened positive affect during the past hour's most positive 
event (i.e., peak positive affect; Morgan et al., 2016). Following pleasurable events, describing the emotional experience of those events to others also enhances positive affect (Rimé, 2009). Notably, positive affect enhancement most strongly occurs during social sharing when close others engage constructively to further discuss the meaning or details of the events (Gable, Reis, Impett, \& Asher, 2004). Emotional closeness also reduces the distressing experiences of daily worry and depressive symptoms among people who highly desire emotional closeness (Flores \& Berenbaum, 2014). Altogether, emotional closeness is an important socialaffective experience that enhances social relationship quality, promotes well-being, and elicits positive affect.

\section{The role of social brain regions in social reward, emotional closeness, and positive affect during social interactions}

Social brain regions may play a role in how adolescents' social interactions elicit positive affect. There is evidence that perceptions of emotional closeness may be associated with response in social brain regions. For example, emotional closeness modulates response in social brain regions implicated in empathy (e.g., temporal poles) when viewing a friend experiencing social rejection (Beeney, Franklin, Levy, \& Adams, 2011). Although this finding was in the context of a negative peer event, it is plausible that emotional closeness may also be related to neural response to social rewards (e.g., positive peer feedback). Also, this finding is an example of emotional closeness having an impact on social brain functioning. It is worth noting, though, that the association between emotional closeness and social brain regions may be bidirectional, such that function in neural social circuitry may also facilitate the experience of emotional closeness (e.g., recruiting brain regions key to empathy may contribute to someone feeling emotionally close to another person). Also, neural response in social brain regions may reflect interpersonally relevant personality traits (e.g., sociability, agreeableness) and trait-like tendencies in how an individual processes socially rewarding contexts (e.g., engaging in greater social processing), which may alter an affective response to a positive social interaction. Thus, individual differences in neural response in social brain regions to objective social rewards may moderate the positive association between emotional closeness and positive affect in adolescents' everyday lives.

\section{The present study}

The present study examined the association of adolescents' neural response to social reward with their experience of emotional closeness and positive affect in natural settings. The primary hypothesis was that adolescents who demonstrate greater response in social reward circuitry will also experience greater emotional closeness in their everyday lives. Although the tendency to experience greater emotional closeness during social interactions may include factors that are not socially specific (e.g., high trait positive affect), they may also include socially specific factors (e.g., high valuing of and experience/ comfort with emotionally intimate interactions). Thus, individual differences in emotional closeness may be distinctly related to social reward despite significant overlap in the circuitry processing social and monetary reward (Izuma et al., 2008). Although the focus of the present study is on social reward, we tested an exploratory hypothesis that emotional closeness would be specifically associated with neural response to social but not monetary reward. The present study was not designed to test this exploratory hypothesis but provided a preliminary means to address it, and the intention of including analyses with monetary reward task was to help inform future studies testing this hypothesis. The secondary hypothesis of the present study was that function in regions identified in the initial hypothesis - that is, regions showing an association between emotional closeness and neural response to social reward-will moderate the association between naturalistic emotional closeness and positive affect (both concurrent positive affect and future peak positive affect), such that the associations between emotional closeness and positive affect will be stronger among those with greater neural response than those with lower neural response to social reward. This moderation is expected, as greater recruitment of social brain regions during social rewards may facilitate greater affective responses to emotionally close interactions.

\section{Method}

\section{Participants}

Participants included in the analyses were 34 typically developing adolescents, ages 14 to 18 years $(M=16.3 \pm 1.5$ years; $65 \%$ female; 3\% Hispanic/Latino; 79\% White, $15 \%$ Black, and $6 \%$ other or multiracial), with no history of psychiatric or serious medical problems, as confirmed by brief interview. Additional participants were excluded due to not participating in the ecological momentary assessment (EMA; $n=8)$, completing fewer than $50 \%$ of the EMA prompts $(n=12)$, not completing the fMRI tasks (due to technical difficulties, claustrophobia, or experiencing a concussion between visits; $n=5$ ), or having fMRI data with insufficient coverage $(n=2)$. The demographics of the excluded participants were not significantly different than included participants, age: $M=16.1 \pm 1.2$ years, $M_{\text {diff }}=0.21, t(59)=0.60, p=.552$; gender: $43 \%$ female, $\chi^{2}(1)=3.48, p=.062$; ethnicity: $0 \%$ Hispanic/Latino, we were unable to run a chi-square test due to there being one 
included participant and zero excluded participants identifying as Hispanic/Latino; race: $63 \%$ White, $26 \%$ Black, and $11 \%$ other or multiracial, $\chi^{2}(2)=2.03, p=.362$. Exclusion was also unrelated to EMA variables, mean closeness, $M_{\text {diff }}=$ $-0.27, t(53)=-1.46, p=.150$; mean positive affect, $M_{\text {diff }}=$ $-0.04, t(54)=-0.22, p=.828 ;$ mean peak happiness, $M_{\text {diff }}=$ $-0.14, t(54)=-1.09, p=.280$; percentage of time with someone, $M_{\text {diff }}=8.2 \%, t(54)=1.68, p=.099$. One participant was excluded from any analyses that included the social reward fMRI task due to being an outlier (i.e., \pm 2 standard deviations from the mean). One participant whose neural response was an outlier on the monetary reward fMRI task and four participants who indicated that they suspected that the monetary reward fMRI task had predetermined outcomes were excluded from analyses that included that task. They were not excluded from other analyses. Thus, 33 participants were included in the primary social reward analyses and, 29 participants were included in the exploratory monetary reward analyses. Given that there is not a widely accepted gold standard to conduct a power analysis for multilevel models, we conducted a power analysis for a related statistical approach (i.e., repeatedmeasures ANOVA). Using G*Power Version 3.1.9.2 (Faul, Erdfelder, Lang, \& Buchner, 2007), we found that a sample size of 32 with at least 14 observations for each participant provides $80 \%$ power to detect a medium-sized effect of a within-between interaction at $\alpha<.05$. Participants completed an EMA protocol, a social-reward fMRI task, and a monetaryreward fMRI task. The University of Pittsburgh Institutional Review Board approved all research procedures, and written informed consent was obtained from each participant and a parent/guardian.

\section{Measures}

Ecological momentary assessment (EMA) Participants received 28 phone calls to cellular phones over a 2-week period (two calls on Thursdays, Fridays, and Mondays after school hours and four calls throughout the day on Saturdays and Sundays, at semirandom times; see Fig. 1 for an illustration of the nested nature of the EMA data). They responded to a variety of questions related to their affect, behavior, and social context. The included participants (who all completed at least $50 \%$ of the prompts) completed an average of $72.7 \%$ of the EMA prompts $(M=20.35, S D=3.66$, range $=14-27)$.

Positive affect Current Positive Affect: In each phone call, participants responded to four questions from the Positive and Negative Affective Schedule for Children (PANAS-C; Laurent et al., 1999) that assessed positive affect at the time the phone rang (i.e., "How would you rate how [happy/cheerful/interested/excited] you were?") using a 5-point Likert scale $(1=$ not at all $; 5=$ extremely; 2 -week mean positive affect: $M=2.82, S D=0.70)$. Peak Happiness: Participants were also asked to "Think about the most enjoyable or happy time in the past hour." They then responded to the question of "At the best point, how happy did you feel?" on a 5-point Likert scale ( $1=$ not at all; $5=$ extremely; 2 -week mean peak happiness: $M=3.89, S D=0.46$ ).

Emotional closeness In each phone call, participants were asked whether they were interacting with someone when they received the phone call and with whom. Participants reported interacting with someone on an average of $40.5 \%(S D=16.2$; range $=11.1 \%-87.5 \%)$ of their completed prompts. If they were interacting with someone, they were also asked to rate "How close or connected did you feel to [person they were interacting with]?" on a 5 -point Likert scale $(1=$ not at all; $5=$ extremely; 2-week mean emotional closeness: $M=3.84, S D=$ 0.65 ). Who they were interacting with included friends (37.7\% of the prompts they endorsed interacting with someone; emotional closeness: $M=4.09, S D=0.86$ ), child/ adolescent family members $(20.6 \% ; M=3.89, S D=0.94)$, adult family members $(30.3 \% ; M=3.59, S D=0.98)$, family in general $(7.2 \% ; M=4.13, S D=0.99)$, and others $(4.2 \% ; M=$ $3.37, S D=1.21)$.

Social-reward fMRI task Participants completed an adapted version of the likeability task (see Fig. 1) developed by Davey et al. (2010); see Healey et al. (2014) for further details. In this block-design task, participants received positive social feedback in the form of being liked by other adolescents. In the first visit, participants rated 32 (50\% female) peer photographs based on how much they thought they would like the person depicted on a 9-point Likert scale $(1=$ not at all; $9=$ very much). They had their own picture taken and were told that these peers would rate their picture. At the neuroimaging assessment $(M=5.88$ weeks later, $S D=9.13$ weeks, median = 3.79 weeks; $M=3.94$ weeks later, $S D=2.80$ weeks, when excluding two outliers; number of weeks between visits was not associated with neural response to social reward, $\rho=-.15$, $p=.418$ ), they viewed the same peer photographs in the scanner with feedback that the peer rated them highly (positive feedback) or did not rate them yet (neutral feedback). Photographs were rank ordered within each gender for each participant based on the ratings they made in the first visit. The top and bottom four photographs in each gender were categorized as "highest rated" and "lowest rated," respectively. The remaining photographs were rated in the middle. Pseudofeedback was presented, such that participants received positive feedback from photographs that they rated highest (i.e., highly rewarding feedback) and lowest (i.e., less rewarding feedback) within each gender. Participants received neutral feedback from photographs that they rated in the middle. Each photograph had a green (positive) or white (neutral) background to indicate feedback. Each photograph was presented three times within eight blocks of 12 stimuli each. 


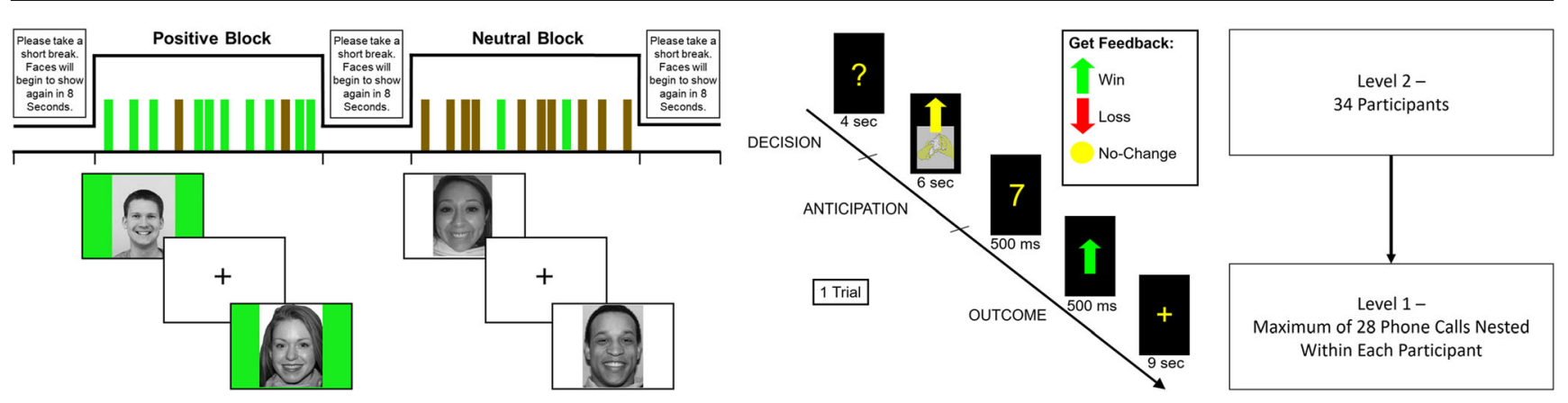

Fig. 1 Illustrations of the block-design social reward task (modified from Healey et al., 2014), the event-related design monetary reward task (taken from Nusslock et al., 2012), and the nested nature of the ecological momentary assessment protocol. (Color figure online)

There were two primarily high positive blocks, two primarily low positive blocks, and four primarily neutral blocks. Blocks included 10 stimuli with feedback of their respective valence (i.e., high positive, low positive, or neutral) and two stimuli of the opposite type (i.e., neutral feedback in positive blocks, high or low positive feedback in neutral blocks) to reduce predictability and habituation. Participants were unaware that the feedback would be presented in blocks. Each photograph was presented for $3 \mathrm{~s}$ with a jittered intertrial crosshair display between stimuli $(1,3,5$, or $7 \mathrm{~s})$. The interblock interval of a "please take a break" message (i.e., "Please take a short break. Faces will begin to show again in 8 seconds.") was $8 \mathrm{~s}$. Task duration was 12.8 minutes. After the scan, participants completed ratings in which they recalled how good they felt when they saw each of the stimulus photographs on a 9-point Likert scale $(1=$ not good at all; $9=$ very good $)$. When presented for these ratings, stimulus photographs were explicitly grouped by feedback (i.e., positive and neutral) with feedback group order counterbalanced. We grouped stimulus photographs by feedback to orient them to the feedback they received to prevent them from misremembering and providing inaccurate ratings. As expected, participants rated feeling better when getting positive feedback from peers they rated highest $(M=6.00, S D=$ $0.97)$ than peers they rated lowest $\left(M=5.47, S D=0.85 ; M_{\text {diff }}=\right.$ $0.53), t(35)=3.53, p=.001$, or when getting neutral feedback $\left(M=4.97, S D=1.03 ; M_{\text {diff }}=1.03\right), t(35)=6.10, p<.001$. Participants did not report significant differences in feeling better from getting feedback from either gender.

Monetary-reward fMRI task The fMRI monetary-reward task (see Fig. 1) used was a card-guessing paradigm adapted from Delgado, Nystrom, Fissell, Noll, and Fiez (2000) to include both anticipation and outcome conditions (Nusslock et al., 2012). In this event-related paradigm, each trial included both an anticipation and an outcome period, and participants received win, loss, or no-change feedback for each trial. Participants were told that they would receive $\$ 1$ for each win, lose 50 cents for each loss, and experience no earnings change for neutral outcomes. Participants were unaware of the fixed outcomes.
Trials were presented in pseudorandom order with predetermined outcomes. During each trial, participants had 4 seconds to guess, through button press, whether the value of a visually presented card with a possible value of 1-9 was higher or lower than 5 (index and middle finger, respectively). Afterward, the trial type (reward or loss) was presented visually for $6 \mathrm{~s}$ (anticipation). This was followed by the "actual" numerical value of the card (500 ms); outcome feedback (a green upward-facing arrow for win, a red downward-facing arrow for loss, or a yellow circle for neutral feedback; 500 $\mathrm{ms}$ ); and a crosshair presented for $9 \mathrm{~s}$ (outcome period included the presentation of value of card, outcome feedback, and the first 6 seconds of the crosshair; baseline consisted of the last 3 seconds of the crosshair presentation). There were six trials of each outcome (i.e., win, loss, no win, no loss). Response time and percentage of responses were not significantly associated with demographic or EMA variables. During debriefing, all but four participants stated that they understood the task, thought that outcomes were due to chance, and found the task engaging.

\section{FMRI acquisition and preprocessing}

Participants were scanned using a Siemens 3T Trio scanner at the University of Pittsburgh Magnetic Resonance Research Center (MRRC). MPRAGE structural images were acquired with high-resolution T1-weighted images with 1-mm isometric voxels $(\mathrm{TR} / \mathrm{TE} / \mathrm{flip}$ angle $=2,300 \mathrm{~ms} / 2.98 \mathrm{~ms} / 9 ; \mathrm{FOV}=$ $256 \times 240 ; 1.2-\mathrm{mm}$ slice; 160 slices; $256 \times 240$ matrix; 1 Nex). Functional blood-oxygen-level-dependent (BOLD) images were acquired using gradient echo planar imaging (EPI) sequences: 39 oblique axial slices (3.1-mm thick, $0-\mathrm{mm}$ gap) oriented to the AC-PC line $(\mathrm{TR} / \mathrm{TE}=2,000 \mathrm{~ms} / 30 \mathrm{~ms} ; \mathrm{FOV}=$ $205 \times 205$; matrix $=64 \times 64)$. A reference EPI scan acquired prior to fMRI data collection was visually inspected for artifacts and signal quality.

Statistical Parametric Mapping software, version 8 (SPM8; http://www.fil.ion.ucl.ac.uk/spm) was used to perform fMRI analyses. Images for each subject were realigned, motioncorrected, and high-pass temporally filtered with a cutoff of 
$128 \mathrm{~s}$. High-motion volumes $(\geq 2 \mathrm{~mm})$ were adjusted using ART (http://gablab.mit.edu/index.php/software). The means $(S D)$ for motion of this sample for the social-reward task were $x=0.17 \mathrm{~mm}(0.12), y=0.38 \mathrm{~mm}(0.51), z=0.74 \mathrm{~mm}$ $(0.90)$, roll $=0.58^{\circ}(0.57)$, pitch $=0.31^{\circ}(0.24)$, and yaw $=$ $0.20^{\circ}(0.17)$. The means $(S D)$ for motion of this sample for the monetary-reward task were $x=0.16 \mathrm{~mm}(0.14), y=0$. $28 \mathrm{~mm}(0.29), z=0.54 \mathrm{~mm}(0.54)$, roll $=0.53^{\circ}(0.52)$, pitch $=0.22^{\circ}(0.19)$, and yaw $=0.23^{\circ}(0.27)$. The mean functional image was coregistered with the high-resolution 3-D anatomic image, normalized to MNI (Montreal Neurological Institute) space, and spatially smoothed (Gaussian kernel 6.0 mm FWHM).

\section{Data analytic strategy}

Neural response to social and monetary reward and mean emotional closeness Neural response to social reward was determined by individual-level analyses contrasting brain activity during receipt of high positive feedback (i.e., mutual liking) compared with blocks of neutral feedback (high positive $>$ neutral feedback), as this contrast reflects high social reward. Block periods included both feedback stimuli and crosshair interstimulus intervals. Neural response to monetary reward focused on consummatory reward ("win" outcome > "no win" outcome contrast) and did not include anticipatory reward, given that the social reward task did not have an equivalent anticipatory period. Outcome periods included presentation of value of card, outcome feedback, and the first 6 seconds of the crosshair; baseline periods consisted of $3 \mathrm{sec}-$ onds of the crosshair following the outcome period. These individual-level analyses included "please take a break" messages (i.e., "interblock interval" for social reward) and some crosshair displays (i.e., "baseline" for monetary reward) as regressors of noninterest; they did not include any other regressors of noninterest. The social-reward and monetaryreward tasks were designed separately and selected for their ability to engage reward circuitry (and social circuitry, in the case of the social-reward task). Although the present study focused on social reward, including the monetary-reward task provided the opportunity to test the primary hypothesis in a monetary-reward task to help inform future studies focused on comparing these two types of reward. We conducted similar but separate regression analyses in SPM8 for each fMRI task with mean EMA-measured emotional closeness as an independent variable and neural response to social or monetary reward as the dependent variable. Age and gender were included as covariates in all analyses considering developmental changes in the social brain network (Burnett et al., 2011) and gender differences in social rewards (Spreckelmeyer et al., 2009). To focus on brain regions related to social processing in general without focusing on a particular type of social process, we masked results based on meta-analytic findings for studies examining the construct "social" in Neurosynth (www.neurosynth.org), a repository of functional neuroimaging masks based on findings across thousands of articles. We used a single forward-inference mask that was generated from 1,000 articles and included brain regions (e.g., mPFC, pSTS, TPJ, VLPFC) that are consistently reported to be activated in articles with frequent use of the term social (see Fig. 2). Given the high overlap between social and reward circuitry, it is not surprising that this mask also included key regions of reward circuitry (e.g., mPFC, striatum, insula, amygdala). We included clusters of at least 50 voxels in the cerebrum. This mask was used to constrain second-level regression analyses to social and reward brain regions. To avoid Type I error, we conducted Monte Carlo simulations using 3dClustSim in AFNI (https://afni.nimh.nih.gov/pub/ dist/doc/program_help/3dClustSim.html) with the autocorrelation function (ACF) estimated by $3 \mathrm{dFWHMx}$, which helps address concerns of previous cluster thresholding techniques (Eklund, Nichols, \& Knutsson, 2016). This procedure estimated that the minimum number of contiguous voxels per cluster (activated at $p_{\text {unc }}<.005$ ) required for a corrected $p<.05$ in a mask of 23,917 voxels was 150 voxels. Principal eigenvariates for clusters reaching significance in second-level analyses were extracted for use in multilevel modeling.

\section{Neural response to social reward as moderator of within-} person association between emotional closeness and concurrent positive affect We used multilevel modeling to examine whether emotional closeness was associated with concurrent positive affect and whether brain regions identified in the first set of analyses described above moderated the within-person association between emotional closeness and concurrent positive affect. Multilevel modeling is a ubiquitous technique to analyze nested data, such as EMA data, in which repeated measurements of participant responses are nested within the participants. Multilevel modeling is useful to analyze EMA data because it does not assume that data points are independent and can handle missing data points (Snijders \& Bosker, 2011). In addition, it allows the ability to examine whether the association between two within-person variables (Level 1; e.g., momentary emotional closeness and positive affect) is moderated by a between-person variable (Level 2; e.g., neural response to social reward measured at one time point). For each of the multilevel modeling analyses, we used the MIXED procedure of the SAS 9.4 software. We report parameter estimates with standard errors. We included random intercepts in each model (which means that intercepts are allowed to be different for each participant) and used unstructured covariance matrices (which means that each variance and covariance in the model is estimated from the data without assuming that variances or covariances are equal). Random 


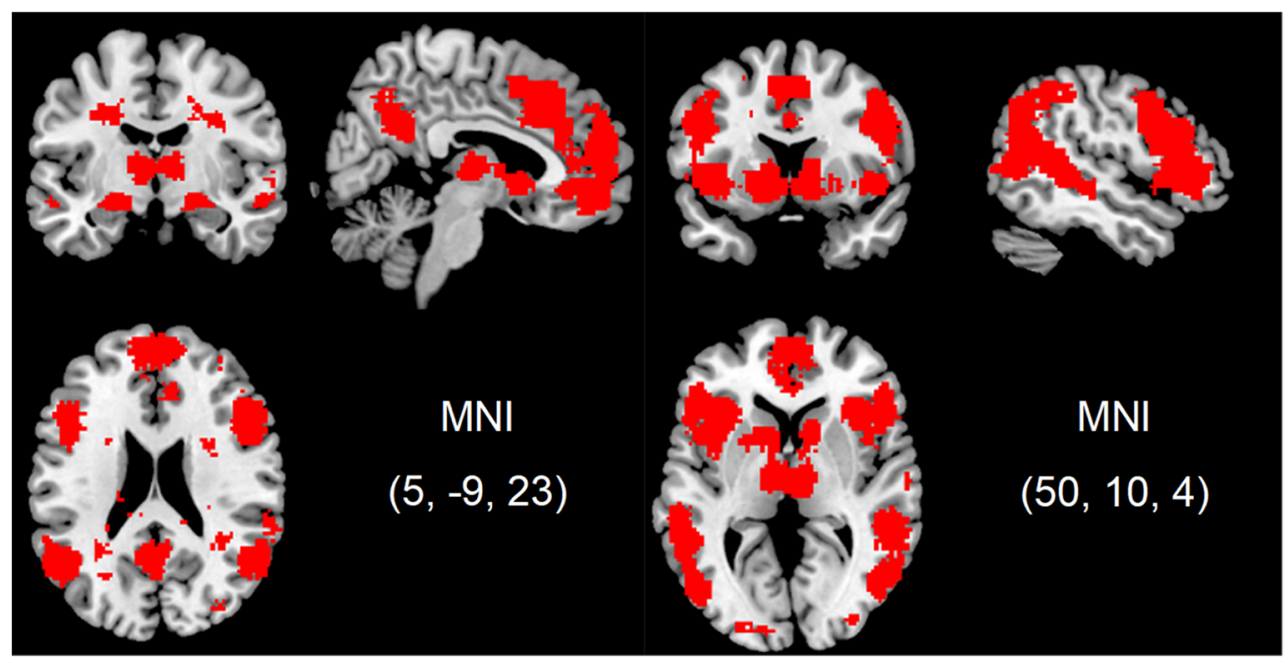

Fig. 2 The Neurosynth-derived "social" mask used in analyses testing the association between momentary emotional closeness and neural response to social and monetary reward. Regions in this mask include the temporoparietal junction (TPJ), posterior superior temporal sulcus (pSTS), inferior parietal lobule (IPL), precuneus, posterior cingulate cortex (PCC), supramarginal gyrus, angular gyrus, parahippocampal gyrus,

slopes (which means that slopes are allowed to be different for each participant) were not included in the models due to final Hessian and estimated G matrix not being positive definite when they were included. We participant-centered within-person predictor variables and group-centered between-person predictor variables. Gender $($ male $=0$; female $=1)$ was dummy coded. A representative model is shown below $(\gamma$ represents coefficient estimates; $\gamma_{30}$ represents the coefficient estimate for the main effect of concurrent closeness; $\gamma_{31}$ represents the coefficient estimate for the Brain Region $\times$ Concurrent Closeness interaction; $R_{\mathrm{ij}}$ and $U_{0 \mathrm{j}}$ are error terms). Time refers to time of day in hours and PA refers to concurrent positive affect.

Level $1: \mathrm{PA}_{(\mathrm{t}) \mathrm{ij}}=\beta_{0 \mathrm{j}}+\beta_{1 \mathrm{j}}\left(\text { Time }_{\mathrm{t}}\right)_{\mathrm{ij}}+\beta_{2 \mathrm{j}}\left(\text { Previous }_{\mathrm{PA}} \mathrm{t}_{\mathrm{t}-1}\right)_{\mathrm{ij}}$

$$
+\beta_{3 \mathrm{j}}\left(\text { Concurrent Closeness } \mathrm{t}_{\mathrm{ij}}+R_{\mathrm{ij}}\right.
$$

Level $2: \beta_{0 \mathrm{j}}=\gamma_{00}+\gamma_{01}(\text { Gender })_{\mathrm{j}}+\gamma_{02}(\text { Age })_{\mathrm{j}}$

$$
\begin{aligned}
& +\gamma_{03}(\text { Brain Region })_{\mathrm{j}}+\mathrm{U}_{0 \mathrm{j}} \\
\beta_{1 \mathrm{j}}= & \gamma_{10} \\
\beta_{2 \mathrm{j}}= & \gamma_{20} \\
\beta_{3 \mathrm{j}}= & \gamma_{30}+\gamma_{31}{\text { (Brain Region })_{\mathrm{j}}}
\end{aligned}
$$

Neural response to social reward as moderator of withinperson association between emotional closeness and future peak happiness Prospective analyses were conducted to test whether previous emotional closeness predicted peak happiness reported at the next call a few hours later and whether brain regions identified in the first set of analyses moderated this association. A representative model is shown below $\left(\gamma_{30}\right.$ represents the coefficient estimate for the main effect of ventrolateral prefrontal cortex (VLPFC), dorsolateral prefrontal cortex (DLPFC), medial prefrontal cortex (mPFC), orbital frontal cortex (OFC), anterior cingulate cortex (ACC), insula, caudate, putamen, ventral striatum, amygdala, thalamus, globus pallidus, supplementary motor area, and portions of the occipital lobe. (Color figure online)

previous closeness; $\gamma_{31}$ represents the coefficient estimate for the Brain Region $\times$ Previous Closeness interaction).

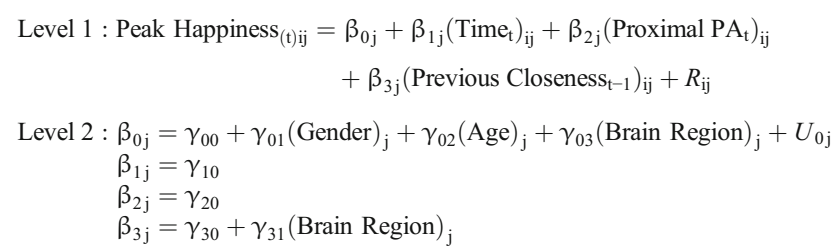

\section{Results}

\section{Between-person associations between neural response to social and monetary reward and mean emotional closeness}

We conducted similar but separate regression analyses for each fMRI reward task, with mean EMA-measured emotional closeness as an independent variable and neural response to social or monetary reward as the dependent variable. Age and gender were included as covariates. To focus on brain regions related to social processing, we masked results based on metaanalytic findings for studies examining the construct "social." There was a positive association between mean emotional closeness and neural response to social reward in the right pSTS/TPJ region (see Table 1 and Fig. 3). No brain regions were found to have a significant negative association. There were no significant age or gender differences in neural response to social reward. As anticipated, there were no significant associations between neural response in any brain region to consummatory monetary reward and mean emotional 
Table 1 Region with a significant between-person positive association between mean EMA emotional closeness and social reward BOLD contrast response (high positive $>$ neutral feedback)

\begin{tabular}{llllll}
\hline Brain region & $\begin{array}{l}\text { Number } \\
\text { of voxels } \\
\text { in cluster }\end{array}$ & $\begin{array}{l}\text { Max T score } \\
\text { at peak voxel }\end{array}$ & $\begin{array}{l}\text { MNI coordinates } \\
\text { of peak voxel } \\
(x, y, z)\end{array}$ \\
\hline $\begin{array}{l}\text { Right posterior superior } \\
\text { temporal sulcus/ } \\
\text { temporoparietal junction }\end{array}$ & 153 & 3.83 & 50 & -54 & 6 \\
\hline
\end{tabular}

Note. Voxels were thresholded at $p<.005$. The findings reported here are significant at corrected $p<.05$ using Monte Carlo simulations

closeness. Furthermore, to test whether there was a significant difference between social and monetary reward in the correlation between right $\mathrm{pSTS/TPJ}$ neural response and mean emotional closeness, we extracted principal eigenvariates from both tasks for the significant cluster found in the right pSTS/TPJ region (see Table 1 and Fig. 3). ${ }^{1}$ Although the correlation with response to the social-reward task was significant $(r=.48, p=.009)$ and the correlation with response to the monetary-reward task was not $(r=.31, p=.105)$, the two correlations were not significantly different when using Fisher $r$-to- $z$ transformations $(z=0.73, p=.463)$.

\section{Neural response to social reward as moderator of concurrent within-person association between emotional closeness and positive affect}

Using the right pSTS/TPJ cluster identified in the previous analysis, we extracted principal eigenvariates from the social-reward and monetary-reward tasks to examine whether neural response to social or monetary reward moderates the within-person relation between emotional closeness and concurrent positive affect. ${ }^{2}$ As expected, emotional closeness was positively associated with concurrent positive affect (see Table 2). In the social reward and concurrent positive affect

\footnotetext{
${ }^{1}$ As an alternative to extracting principal eigenvariates from the significant right pSTS/TPJ cluster found in the social reward analyses, we also tried using a meta-analytic functional mask (2,226 voxels) from Neurosynth.org by combining the primary right clusters from masks generated by using the terms pSTS (based on 73 articles) and temporoparietal junction (based on 130 articles). The results were similar when using the meta-analytic functional mask. Although the correlation with response to the social-reward task was significant $(r=.38, p=.046)$ and the correlation with response to the monetary reward task was not $(r=.05, p=.788)$, the two correlations were not significantly different when using Fisher $r$-to- $z$ transformations $(z=1.08, p=.282)$.

${ }^{2}$ We also tried conducting the multilevel models with principal eigenvariates of right pSTS/TPJ response using the meta-analytic functional mask described in footnote 1. Although right pSTS/TPJ response to social reward did not significantly moderate the association between emotional closeness and concurrent positive affect, $\gamma_{31}=-0.13, S E=0.09, t(194)=-1.34, p=.182$, it did significantly moderate the association between emotional closeness and future peak happiness, $\gamma_{31}=0.20, S E=0.09, t(161)=2.15, p=.033$. Right pSTS/ TPJ response to monetary reward did not significantly moderate either association, concurrent positive affect: $\gamma_{31}=-0.31, S E=0.20, t(162)=-1.55, p=$ .123 , future peak happiness: $\gamma_{31}=0.15, S E=0.23, t(140)=0.63, p=.531$.
}
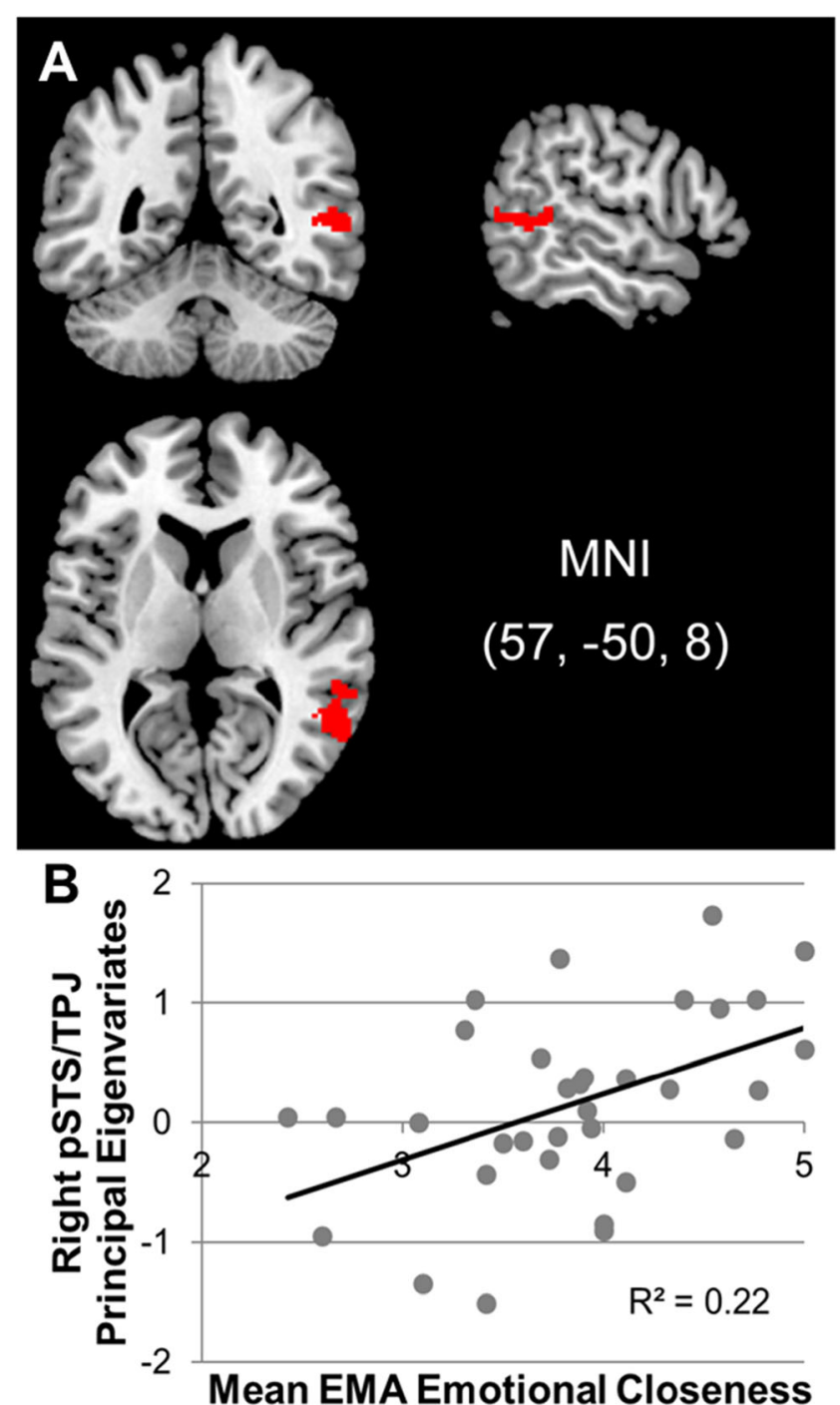

Fig. 3 a Brain region (right posterior superior temporal sulcus/ temporoparietal junction [pSTS/TPJ]) with a positive association between mean ecological momentary assessment (EMA) emotional closeness and neural response to social reward (high positive $>$ neutral feedback). b Scatterplots of between-person associations between EMA emotional closeness and neural response to social reward. (Color figure online)

model, neural response to social reward moderated the concurrent association between emotional closeness and positive affect. ${ }^{3}$ The positive association between emotional closeness

\footnotetext{
${ }^{3}$ We considered including mean level of closeness at Level 2 but decided to not include it given that the moderation hypotheses were focused on withinperson differences in emotional closeness rather than between-person differences in emotional closeness. When including mean level of closeness at Level 2, mean closeness was significantly related to positive affect, social reward: $\gamma_{04}=0.64, S E=0.18, t(32.6)=3.47, p=.002$; monetary reward: $\gamma_{04}=0.45$, $S E=0.17, t(30.7)=2.67, p=.012$, and future peak happiness, social reward: $\gamma_{04}=0.59, S E=0.15, t(34.3)=3.87, p=.001 ;$ monetary reward: $\gamma_{04}=0.52$, $S E=0.13, t(34.4)=3.95, p<.001$. The coefficient estimates for Level 1 emotional closeness and the right pSTS/TPJ $\times$ Level 1 emotional closeness interaction did not change in each model.
} 
Table 2 Unstandardized coefficient estimates (and standard errors) for multilevel models examining the moderating roles of right pSTS/TPJ response to social and monetary reward in the associations between emotional closeness and both concurrent positive affect and prospective peak happiness

\begin{tabular}{|c|c|c|c|c|}
\hline & $\begin{array}{r}\text { Concurrent } \\
\text { Positive Affect } \\
\end{array}$ & $\begin{array}{c}\underline{\text { Prospective Peak }} \\
\underline{\text { Happiness }}\end{array}$ & $\begin{array}{r}\text { Concurrent } \\
\text { Positive Affect } \\
\end{array}$ & $\begin{array}{c}\underline{\text { Prospective Peak }} \\
\underline{\text { Happiness }}\end{array}$ \\
\hline \multicolumn{5}{|l|}{ Level 1 Variables } \\
\hline Time, $\gamma_{10}$ & $0.01(0.02)$ & $0.03(0.02)$ & $0.00(0.02)$ & $0.04(0.02)^{\dagger}$ \\
\hline $\begin{array}{l}\text { Previous Positive Affect, } \\
\gamma_{20}\end{array}$ & $0.08(0.07)$ & & $0.06(0.08)$ & \\
\hline $\begin{array}{l}\text { Proximal Positive Affect, } \\
\gamma_{20}\end{array}$ & & $0.48(0.07)^{* * *}$ & & $0.45(0.08)^{* * *}$ \\
\hline Concurrent Closeness, $\gamma_{30}$ & $0.37(0.05)^{* * *}$ & & $0.22(0.06)^{* * *}$ & \\
\hline Previous Closeness, $\gamma_{30}$ & & $0.20(0.06)^{* * *}$ & & $0.24(0.06)^{* * *}$ \\
\hline Level 2 Variables & & & & \\
\hline Intercept, $\gamma_{00}$ & $3.08(0.20)^{* * *}$ & $3.87(0.17)^{* * *}$ & $3.20(0.20)^{* * *}$ & $3.89(0.18)^{* * *}$ \\
\hline Gender, $\gamma_{01}$ & $-0.26(0.23)$ & $-0.11(0.19)$ & $-0.37(0.25)$ & $-0.17(0.20)$ \\
\hline Age, $\gamma_{02}$ & $0.17(0.08)$ & $0.04(0.07)$ & $0.17(0.08)^{*}$ & $0.04(0.07)$ \\
\hline Right pSTS/TPJ, $\gamma_{04}$ & $0.09(0.15)$ & $0.20(0.13)$ & $0.60(0.39)$ & $0.31(0.34)$ \\
\hline Right $\mathrm{pSTS} / \mathrm{TPJ} \times$ & $-0.15(0.08)^{*}$ & $0.20(0.09)^{*}$ & $-0.19(0.22)$ & $0.05(0.21)$ \\
\hline
\end{tabular}

Closeness, $\gamma_{31}$

Note. Gray cells indicate that the predictor variable was not included in the model.

$* p<.05 . * * * p<.001 .{ }^{\dagger} p<.10$

and concurrent positive affect was unexpectedly greater among individuals who demonstrated lower right pSTS/ TPJ response to social reward than those who demonstrated higher right pSTS/TPJ response (see Fig. 4). A followup simple slopes test demonstrated that the positive association between emotional closeness and concurrent positive affect was significant when the principal eigenvariate of right pSTS/TPJ was less than 1.17 (i.e., 1.52 standard deviations above the mean in the present sample). Thus, the association was significant at both one standard deviation above, $t(194)=3.44, p=.001$, and one standard deviation below, $t(194)=5.82, p<.001$, the mean of right pSTS/TPJ response. It is worth noting that this simple slopes test does not require the sample to be divided into 


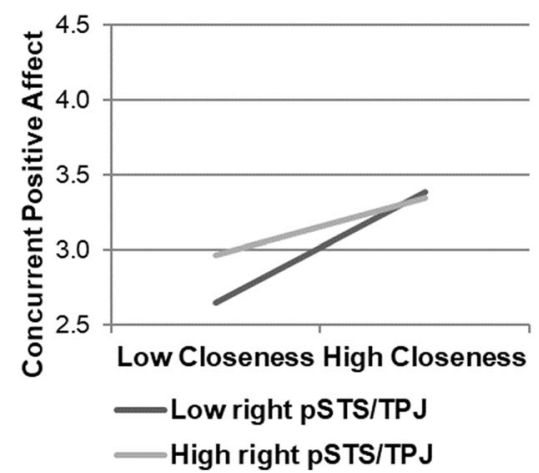

Fig. 4 Graphs based on multilevel models demonstrating the ecological momentary assessment (EMA) within-person association between emotional closeness and concurrent positive affect or future peak happiness at

groups (see Preacher, Curran, \& Bauer, 2006; http://www. quantpsy.org/interact/hlm2.htm).

As expected, emotional closeness predicted future peak happiness (see Table 2), and right pSTS/TPJ response to social reward moderated this association (see Fig. 4). Follow-up simple slopes tests (see Preacher et al., 2006) demonstrated that there was a significant positive association between emotional closeness and future peak happiness when the principal eigenvariate of right $\mathrm{pSTS} / \mathrm{TPJ}$ was at least -0.38 (i.e., 0.49 standard deviations below the mean in the present sample). Thus, this association was significant at one standard deviation above the mean of right $\mathrm{pSTS} / \mathrm{TPJ}$ response to social reward, $t(164)=3.69$, $p<.001$. However, the association was not significant at one standard deviation below the mean of right $\mathrm{pSTS} / \mathrm{TPJ}$ response, $t(164)=0.51, p=.614$. Right $\mathrm{pSTS} / \mathrm{TPJ}$ response to monetary reward did not moderate either association (see Table 2).

\section{Discussion}

To better understand adolescents' individual differences in experiencing positive affect related to rewarding social interactions, the present study examined whether neural response to social reward is (1) associated with mean levels of real-world emotional closeness - an important social experience that can elicit positive affect in everyday lifeand (2) a moderator of the within-person association between real-world emotional closeness and positive affect. Adolescents who exhibited greater neural response to social reward in a key social processing region (i.e., right pSTS/TPJ) generally experienced greater emotional closeness in their everyday lives. In addition, right pSTS/TPJ response to social reward moderated the within-person association between naturalistic emotional closeness and both concurrent positive affect and future peak happiness.

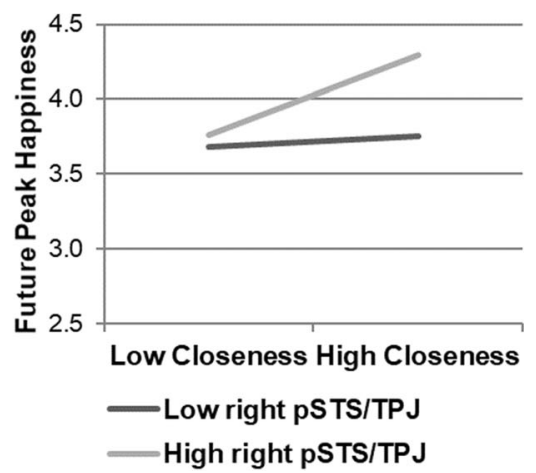

low and high right $\mathrm{pSTS} / \mathrm{TPJ}$ contrast BOLD response (high positive > neutral feedback), with low and high defined as one standard deviation below and above the mean principal eigenvariate, respectively

The nature of the moderation was different, however, between concurrent and prospective associations. Surprisingly, adolescents with lower right pSTS/TPJ response demonstrated a stronger positive association between emotional closeness and concurrent positive affect than those with higher right $\mathrm{pSTS} / \mathrm{TPJ}$ response. In contrast, emotional closeness was associated with future peak happiness among adolescents with higher right pSTS/TPJ response but not among those with lower right pSTS/TPJ response. Thus, adolescents with higher right pSTS/TPJ response to social reward seem to experience greater emotional closeness in their everyday life and have a sustained affective benefit from emotional closeness despite having a tempered immediate affective benefit (i.e., they have a relatively weak affective benefit in the short term but a relatively strong affective benefit in the long term).

\section{Between-person association between neural response to social reward and emotional closeness}

The lateralized findings for $\mathrm{pSTS} / \mathrm{TPJ}$ response are consistent with previous research, where the right $\mathrm{pSTS} / \mathrm{TPJ}$ is implicated in social processes, including mentalizing - a construct that generally includes theory of mind, perspective taking, and cognitive empathy (Burnett et al., 2011; Krall et al., 2016). Right pSTS is more specifically implicated in face detection (Allison, Puce, \& McCarthy, 2000) and eye gaze (Puce, Allison, Bentin, Gore, \& McCarthy, 1998), perception of biological motion (Saygin, 2007), and decoding social gestures to predict others' action or intent (Haxby, Hoffman, \& Gobbini, 2000; Morris, Pelphrey, \& MCCarthy, 2005; Saxe, Xiao, Kovacs, Perrett, \& Kanwisher, 2004). Right TPJ is also suggested to play a role in reorientation of attention to both social and nonsocial unexpected stimuli (Decety \& Lamm, 2007). One possibility is that adolescents with greater emotional closeness may more readily engage social processing circuitries in social situations. Further, these adolescents may 
also typically engage in these processes in their everyday lives during positive social interactions. In fact, as adolescents develop and transition to adulthood, they tend to demonstrate greater consideration of others and shift from exhibiting greater $\mathrm{mPFC}$ activation to greater TPJ activation during social decision-making (Crone \& Dahl, 2012). This could reflect greater other orientation, enhanced perspective taking, and stronger self-other identification. These social cognitive advances could provide a foundation for sophisticated social processing (and adaptive functioning) in adulthood. Future longitudinal studies examining the association between neural response to social reward and emotional closeness may find that emotional closeness may play an important role in the developmental change from greater $\mathrm{mPFC}$ to greater TPJ activation during social situations over the course of adolescence.

Potentially, engaging in social processing during socially rewarding situations may help facilitate experiencing greater emotional closeness. For example, greater engagement in social processing may facilitate conceptualizing the interaction as a shared experience. This may enhance the process of incorporating others as part of the self, which might contribute to feelings of emotional closeness (Aron, Aron, Tudor, \& Nelson, 1991). Although this possibility cannot be tested in the present study, future behavioral studies could help address this possibility by manipulating the use of social processes during a socially rewarding task and measuring emotional closeness and the incorporation of the other as part of the self during the task.

It is worth highlighting that research on reward does not commonly include two classes of reward within the one study, despite the importance of understanding whether effects observed generalize across different classes of rewarding stimuli. Including both social reward and monetary reward, which is a more generic form of reward that is widely used in neuroimaging research, in the present study demonstrated that neural response to social - but not monetary - reward is related to experiencing greater naturalistic emotional closeness. However, the present study did not find a significant difference between social and monetary reward in terms of their correlations between emotional closeness and right pSTS/ TPJ neural response. It is also worth noting though that there are notable differences between the social and monetary reward fMRI tasks used in the present study. For example, the social-reward task used a block design and the monetaryreward task used an event-related design. Thus, the finding that naturalistic emotional closeness is related to social but not monetary reward should be considered preliminary and suggests that it could beneficial for future studies to compare correlates of social and monetary reward using similarly designed tasks. Although there is overlap in reward circuitry activated by social and nonsocial rewards (Izuma et al., 2008), these preliminary findings suggest that there may be components of emotional closeness that are relevant to social but not to nonsocial rewards. For example, valuing emotionally intimate experiences and having experience and comfort with emotional closeness may be related to neural response to social but not to nonsocial rewards.

\section{Right pSTS/TPJ response to social reward moderated within-person associations between emotional closeness and positive affect}

Whereas those with low right pSTS/TPJ response may be more emotionally reactive to the experience of emotional closeness in the immediate moment, those with high right pSTS/TPJ may build on the experience of emotional closeness to achieve a higher peak happiness hours later. Given that the right pSTS/TPJ is associated with social processing, the moderating role of right pSTS/TPJ suggests the potential importance of social processing in the affective benefits of emotional closeness. One possible explanation worthy of further investigation is that mentalizing may enhance the quality of an emotionally close interaction as it progresses by facilitating social competence (Davis, 1983) and prosocial behaviors (Eisenberg \& Miller, 1987). Mentalizing may also increase the meaning of the interaction by enhancing the incorporation of the other person into one's own self-concept (Galinsky, Ku, \& Wang, 2005). The combination of increased quality and meaning of an emotionally close social interaction may help explain how adolescents with higher right pSTS/TPJ response - but not adolescents with lower right pSTS/TPJ response - experienced sustained affective benefits from emotional closeness. Given the developmental shift from $\mathrm{mPFC}$ to TPJ activation over the course of adolescence and the transition into adulthood (Crone \& Dahl, 2012), future studies may find that this shift may facilitate experiencing greater sustained affective benefits from emotional closeness over the course of adolescence.

\section{Strengths, limitations, and future directions}

One notable strength of the present study is that it integrated fMRI and EMA methodologies to examine neural aspects of reward and naturalistic aspects of subjective emotional closeness and positive affect. Key advantages of incorporating the fMRI task include contributing a neural level of analysis and measuring social reward neural response to standardized stimuli. Advantages of EMA include its ecological validity, given that participants make ratings in the context of their everyday life, and its ability to capture both concurrent and prospective associations within a day, which helps demonstrate timebased characteristics of an association.

Although greater neural response to social reward in social brain regions suggest increased social processing during social reward, it is important to note that social processing was not measured directly in the present study. Future studies would 
benefit from measuring the spontaneous use of social processing during social reward (e.g., posttask self-report questionnaire) or manipulating the use of social-cognitive processes (e.g., instructing the use of mentalizing) during social reward. Given that there are developmental changes in both reward and social circuitries during adolescence, future studies should also include participants in other developmental periods (e.g., young adulthood) or a longitudinal design over several years. Incorporating participants with or at risk for psychopathology (e.g., depression) would also be beneficial to elucidate how the present findings may inform the development of psychopathology. Although the present study's relatively modest sample size appears to be sufficient to detect a medium-sized interaction, future studies would be strengthened by having larger sample sizes to further improve the reliability of findings and to be able to detect smaller effect sizes.

\section{Conclusion}

Increased right $\mathrm{pSTS} / \mathrm{TPJ}$ response to social reward was associated with mean emotional closeness and moderated the positive association between emotional closeness and positive affect. Study findings suggest that increased engagement of right $\mathrm{pSTS} / \mathrm{TPJ}$ - a key brain region for social processing during socially rewarding contexts may both facilitate the everyday experience of emotional closeness and support the sustained affective benefits of emotional closeness despite tempering the immediate affective benefits of emotional closeness. Thus, social brain regions (including right $\mathrm{pSTS} / \mathrm{TPJ}$ ) and social-affective experiences (e.g., emotional closeness) appear to play important roles in the experience of positive affect during positive social interactions.

\begin{abstract}
Acknowledgements We would like to thank Barbara Hanusa, Ph.D., for her invaluable statistics consultation, the research assistants who contributed to this study, and the families who participated. This research was supported by a grant from the National Institute on Drug Abuse (DA033612, PI: Forbes). Writing of this manuscript was supported by the Department of Veterans Affairs Office of Academic Affiliations Advanced Fellowship Program in Mental Illness Research and Treatment, the VA Pittsburgh Healthcare System, and the VISN 4 Mental Illness Research, Education and Clinical Center (MIRECC). Authors have no conflicts of interest to declare.
\end{abstract}

Disclaimer The contents of this paper do not represent the views of the U.S. Department of Veterans Affairs or the United States Government.

\section{References}

Allison, T., Puce, A., \& McCarthy, G. (2000). Social perception from visual cues: Role of the STS region. Trends in Cognitive Sciences, 4(7), 267-278.
Aron, A., Aron, E. N., Tudor, M., \& Nelson, G. (1991). Close relationships as including other in the self. Journal of Personality and Social Psychology, 60(2), 241.

Baumeister, R. F., \& Leary, M. R. (1995). The need to belong: Desire for interpersonal attachments as a fundamental human motivation. Psychological Bulletin, 117(3),497-529.

Beeney, J. E., Franklin, R. G. Jr, Levy, K. N., \& Adams, R. B. Jr (2011). I feel your pain: Emotional closeness modulates neural responses to empathically experienced rejection. Social Neuroscience, 6(4), 369 376.

Bjork, J. M., Knutson, B., Fong, G. W., Caggiano, D. M., Bennett, S. M., \& Hommer, D. W. (2004). Incentive-elicited brain activation in adolescents: Similarities and differences from young adults. Journal of Neuroscience, 24(8), 1793-1802.

Blakemore, S. J. (2008). The social brain in adolescence. Nature Reviews Neuroscience, 9(4), 267-277.

Burnett, S., Sebastian, C., Kadosh, K. C., \& Blakemore, S. J. (2011). The social brain in adolescence: Evidence from functional magnetic resonance imaging and behavioural studies. Neuroscience \& Biobehavioral Reviews, 35(8), 1654-1664.

Crone, E. A., \& Dahl, R. E. (2012). Understanding adolescence as a period of social-affective engagement and goal flexibility. Nature Reviews Neuroscience, 13(9), 636-650.

Davey, C. G., Allen, N. B., Harrison, B. J., Dwyer, D. B., \& Yücel, M. (2010). Being liked activates primary reward and midline selfrelated brain regions. Human Brain Mapping, 31(4), 660-668.

Davey, C. G., Yücel, M., \& Allen, N. B. (2008). The emergence of depression in adolescence: Development of the prefrontal cortex and the representation of reward. Neuroscience and Biobehavioral Reviews, 32(1), 1-19.

Davis, M. H. (1983). Measuring individual differences in empathy: Evidence for a multidimensional approach. Journal of Personality and Social Psychology, 44(1), 113-126.

Decety, J., \& Lamm, C. (2007). The role of the right temporoparietal junction in social interaction: How low-level computational processes contribute to meta-cognition. The Neuroscientist, 13(6), 580-593.

Delgado, M. R., Nystrom, L. E., Fissell, C., Noll, D. C., \& Fiez, J. A. (2000). Tracking the hemodynamic responses to reward and punishment in the striatum. Journal of Neurophysiology, 84(6), 30723077.

Eisenberg, N., \& Miller, P. A. (1987). The relation of empathy to prosocial and related behaviors. Psychological Bulletin, 101(1), 91-119.

Eklund, A., Nichols, T. E., \& Knutsson, H. (2016). Cluster failure: Why fMRI inferences for spatial extent have inflated false-positive rates. Proceedings of the National Academy of Sciences, 113(28),7900 7905.

Ernst, M., Nelson, E. E., Jazbec, S., McClure, E. B., Monk, C. S., Leibenluft, E., . . . Pine, D. S. (2005). Amygdala and nucleus accumbens in responses to receipt and omission of gains in adults and adolescents. NeuroImage, 25(4), 1279-1291.

Faul, F., Erdfelder, E., Lang, A. G., \& Buchner, A. (2007). G* Power 3: A flexible statistical power analysis program for the social, behavioral, and biomedical sciences. Behavior Research Methods, 39(2), 175-191.

Flores, L. E., Jr., \& Berenbaum, H. (2014). Desired emotional closeness moderates the prospective relations between levels of perceived emotional closeness and psychological distress. Journal of Social and Clinical Psychology, 33(8), 673-700.

Frith, U., \& Frith, C. D. (2003). Development and neurophysiology of mentalizing. Philosophical Transactions of the Royal Society of London B: Biological Sciences, 358(1431), 459-473.

Forbes, E. E., \& Dahl, R. E. (2012). Altered reward function in adolescent depression: What, when and how? Journal of Child Psychology and Psychiatry, 53(1), 3-15.

Forbes, E. E., Ryan, N. D., Phillips, M. L., Manuck, S. B., Worthman, C. M., Moyles, D. L., . . \& \& Dahl, R. E. (2010). Healthy adolescents' 
neural response to reward: Associations with puberty, positive affect, and depressive symptoms. Journal of the American Academy of Child \& Adolescent Psychiatry, 49(2), 162-172.

Gable, S. L., Reis, H. T., Impett, E. A., \& Asher, E. R. (2004). What do you do when things go right? The intrapersonal and interpersonal benefits of sharing positive events. Journal of Personality and Social Psychology, 87(2), 228-245.

Galinsky, A. D., Ku, G., \& Wang, C. S. (2005). Perspective-taking and self-other overlap: Fostering social bonds and facilitating social coordination. Group Processes and Intergroup Relations, 8(2), 109124

Gross, J. J., \& John, O. P. (2003). Individual differences in two emotion regulation processes: Implications for affect, relationships, and wellbeing. Journal of Personality and Social Psychology, 85(2), 348362.

Gunther Moor, B., Güroğlu, B., Op de Macks, Z. A., Rombouts, S. A., Van der Molen, M. W., \& Crone, E. A. (2012). Social exclusion and punishment of excluders: Neural correlates and developmental trajectories. NeuroImage, 59(1), 708-717.

Guyer, A. E., Choate, V. R., Pine, D. S., \& Nelson, E. E. (2012). Neural circuitry underlying affective response to peer feedback in adolescence. Social Cognitive and Affective Neuroscience, 7(1), 81-92.

Haxby, J. V., Hoffman, E. A., \& Gobbini, M. I. (2000). The distributed human neural system for face perception. Trends in Cognitive Sciences, 4(6), 223-233.

Healey, K. L., Morgan, J., Musselman, S. C., Olino, T. M., \& Forbes, E. E. (2014). Social anhedonia and medial prefrontal response to mutual liking in late adolescents. Brain and Cognition, 89, 39-50.

Hyman, S. E., Malenka, R. C., \& Nestler, E. J. (2006). Neural mechanisms of addiction: The role of reward-related learning and memory. Annual Review of Neuroscience, 29, 565-598.

Izuma, K., Saito, D. N., \& Sadato, N. (2008). Processing of social and monetary rewards in the human striatum. Neuron, 58(2), 284-294.

Kahneman, D., Krueger, A. B., Schkade, D. A., Schwarz, N., \& Stone, A. A. (2004). A survey method for characterizing daily life experience: The day reconstruction method. Science, 306(5702), 1776-1780.

Krall, S. C., Volz, L. J., Oberwelland, E., Grefkes, C., Fink, G. R., \& Konrad, K. (2016). The right temporoparietal junction in attention and social interaction: A transcranial magnetic stimulation study. Human Brain Mapping, 37(2), 796-807.

Laurent, J., Catanzaro, S. J., Joiner, T. E., Jr., Rudolph, K. D., Potter, K. I., Lambert, S., . . . Gathright, T. (1999). A measure of positive and negative affect for children: Scale development and preliminary validation. Psychological Assessment, 11(3), 326-338.

Lin, A., Adolphs, R., \& Rangel, A. (2012). Social and monetary reward learning engage overlapping neural substrates. Social, Cognitive, and Affective Neuroscience, 7(3), 274-281.

Lopes, P. N., Salovey, P., Côté, S., Beers, M., \& Petty, R. E. (2005). Emotion regulation abilities and the quality of social interaction. Emotion, 5(1), 113-118.

Lyubomirsky, S., King, L., \& Diener, E. (2005). The benefits of frequent positive affect: Does happiness lead to success? Psychological Bulletin, 131(6), 803-855.

Monk, C. S., Klein, R. G., Telzer, E. H., Schroth, E. A., Mannuzza, S., Moulton, III, J. L., . . . Blair, R. J. (2008). Amygdala and nucleus accumbens activation to emotional facial expressions in children and adolescents at risk for major depression. American Journal of Psychiatry, 165(1), 90-98.

Morgan, J. K., Lee, G. E., Wright, A. G., Gilchrist, D. E., Forbes, E. E., McMakin, D. L., . . . Silk, J. S. (2016). Altered positive affect in clinically anxious youth: The role of social context and anxiety subtype. Journal of Abnormal Child Psychology, 45(7), 14611472. https://doi.org/10.1007/s10802-016-0256-3

Morris, J. P., Pelphrey, K. A., \& MCCarthy, G. (2005). Regional brain activation evoked when approaching a virtual human on a virtual walk. Journal of Cognitive Neuroscience,17(11), 1744-1752.
Nusslock, R., Almeida, J. R., Forbes, E. E., Versace, A., Frank, E., LaBarbara, E. J., . . . Phillips, M. L. (2012). Waiting to win: Elevated striatal and orbitofrontal cortical activity during reward anticipation in euthymic bipolar disorder adults. Bipolar Disorders, 14(3), 249-260.

Olino, T. M., Silk, J. S., Osterritter, C., \& Forbes, E. E. (2015). Social reward in youth at risk for depression: A preliminary investigation of subjective and neural differences. Journal of Child and Adolescent Psychopharmacology, 25(9), 711-721.

Pfeifer, J. H., \& Blakemore, S. J. (2012). Adolescent social cognitive and affective neuroscience: Past, present, and future. Social, Cognitive, and Affective Neuroscience, 7(1), 1-10.

Plichta, M. M., \& Scheres, A. (2014). Ventral-striatal responsiveness during reward anticipation in ADHD and its relation to trait impulsivity in the healthy population: A meta-analytic review of the fMRI literature. Neuroscience and Biobehavioral Reviews, 38, 125-134.

Preacher, K. J., Curran, P. J., \& Bauer, D. J. (2006). Computational tools for probing interaction effects in multiple linear regression, multilevel modeling, and latent curve analysis. Journal of Educational and Behavioral Statistics, 31, 437-448.

Puce, A., Allison, T., Bentin, S., Gore, J. C., \& McCarthy, G. (1998). Temporal cortex activation in humans viewing eye and mouth movements. Journal of Neuroscience, 18(6), 2188-2199.

Richey, J. A., Rittenberg, A., Hughes, L., Damiano, C. R., Sabatino, A., Miller, S., . . . Dichter, G. S. (2014). Common and distinct neural features of social and non-social reward processing in autism and social anxiety disorder. Social, Cognitive, and Affective Neuroscience, 9(3), 367-377.

Rimé, B. (2009). Emotion elicits the social sharing of emotion: Theory and empirical review. Emotion Review, 1(1), 60-85.

Saxe, R., Xiao, D. K., Kovacs, G., Perrett, D. I., \& Kanwisher, N. (2004). A region of right posterior superior temporal sulcus responds to observed intentional actions. Neuropsychologia, 42(11), 1435-1446.

Saygin, A. P. (2007). Superior temporal and premotor brain areas necessary for biological motion perception. Brain, 130(9), 2452-2461.

Silk, J. S., Stroud, L. R., Siegle, G. J., Dahl, R. E., Lee, K. H., \& Nelson, E. E. (2012). Peer acceptance and rejection through the eyes of youth: Pupillary, eye tracking and ecological data from the chatroom interact task. Social, Cognitive, and Affective Neuroscience, 7(1),93-105.

Smith, A. R., Steinberg, L., Strang, N., \& Chein, J. (2015). Age differences in the impact of peers on adolescents' and adults' neural response to reward. Developmental Cognitive Neuroscience, 11, 75-2.

Snijders, T. A., \& Bosker, R. J. (2011). Multilevel analysis: An introduction to basic and advanced multilevel modeling (2nd ed.). London, UK: SAGE.

Somerville, L. H., Jones, R. M., \& Casey, B. J. (2010). A time of change: Behavioral and neural correlates of adolescent sensitivity to appetitive and aversive environmental cues. Brain and Cognition, 72(1), 124-133.

Spreckelmeyer, K. N., Krach, S., Kohls, G., Rademacher, L., Irmak, A., Konrad, K., . . . Gründer, G. (2009). Anticipation of monetary and social reward differently activates mesolimbic brain structures in men and women. Social, Cognitive, and Affective Neuroscience, $4(2), 158-165$.

Steinberg, L. (2008). A social neuroscience perspective on adolescent risk-taking. Developmental Review, 28(1), 78-106.

Telzer, E. H. (2016). Dopaminergic reward sensitivity can promote adolescent health: A new perspective on the mechanism of ventral striatum activation. Developmental Cognitive Neuroscience, 17, 57-67.

Telzer, E. H., Fuligni, A. J., Lieberman, M. D., \& Galván, A. (2014). Neural sensitivity to eudaimonic and hedonic rewards differentially predict adolescent depressive symptoms over time. Proceedings of the National Academy of Sciences, 111(18), 6600-6605.

Zink, C. F., Tong, Y., Chen, Q., Bassett, D. S., Stein, J. L., \& MeyerLindenberg, A. (2008). Know your place: Neural processing of social hierarchy in humans. Neuron, 58(2), 273-283. 